\title{
MEJORAMIENTO DE CHOCOLATE AMARGO PARA TAZA MEDIANTE EL USO DE LICOR DE CACAO
}

\author{
Gabriela C. Chire F.; Augusta Córdova Rivera \\ Universidad Nacional Mayor de San Marcos (*)
}

\begin{abstract}
RESUMEN
En el presente estudio, se ha procesado el licor de cacao y se ha obtenido un chocolate para taza de variedad amarga con características diferenciales para ser exportado. Mediante el uso de ingredientes permitidos en la Norma Técnica Peruana de Chocolate NTP 208.002 (1999) se han elaborado 13 lotes experimentales, los cuales fueron evaluados por métodos físico químicos y sensoriales; para éste último, la evaluación fue hecha por un panel entrenado (consumidores habituales de chocolate para taza amargo); obteniendo el producto mejorado. Luego se evaluó el tiempo de vida y se realizaron las pruebas en planta obteniendo resultados satisfactorios, el producto se encuentra listo para salir al mercado.
\end{abstract}

Palabras clave: Manteca de cacao, cocoa en polvo, licor de cacao, ingredientes, panel entrenado, chocolate amargo.

\begin{abstract}
SUMMARY
In this research the cocoa mass was processed to obtain, for first time, different bitter cup chocolate available to be exported abroad. According to the use of ingredients which are allowed for food industry in the Norma Técrica Peruana de Chocolate; was formulated 13 different recipes each one was evaluated by physical - chemistry and sensory methods; the sensory evaluation was made by a trained panel (bitter cup chocolate consumers), looking for the better product, in which was choosen. After that, the shelf life, pilot test in factory were assessment, given a satisfactory result, now the product is ready to be launched in the market.
\end{abstract}

Key words: Cocoa butter, cocoa powder, cocoa liquor, ingredients, trained panel, bitter chocolate.

\section{INTRODUCCIÓN}

Por primera vez en el Perú, se ha obtenido un chocolate amargo para taza a partir de licor de cacao con las características físico químicas y sensoriales, exigidas por patrones internacionales y cumpliendo las exigencias de la Norma Técnica Peruana y así poder ser competitivos en el mercado internacional. Se ha considerado como objetivos realizar la obtención de licor de cacao más conveniente, de acuerdo a las normas internacionales, empleando especies peruanas de cacao y sugerir, de acuerdo a esta investigación, los manejos adecuados en el correcto almacenamiento y los parámetros de tiempo y temperatura para el mejor diseño y proceso del producto.

\section{MATERIAL Y MÉTODOS}

Lugar de trabajo. Se realizó en la UNMSM (Facultad de Química e Ingeniería Química, Laboratorio de Química Orgánica).
Materia prima. Se utilizaron muestras de granos de cacao, procedentes de Ayacucho y Cusco, por ser las zonas con mayor producción nacional, fermentadas parcialmente, para obtener una muestra homogénea, después se procesó hasta obtener licor de cacao, que se usa como materia prima en la producción de chocolate amargo para taza según formulas propuestas.

Insumos. Los ingredientes usados en las diferentes formulaciones del chocolate amargo para taza incluyen licor de cacao, espumante vana lata $30 \mathrm{~B}$, espumante vana capa 23B, espumante hyfoama DSN, café tostado molido, emulsificante lecitina de soya, emulsificante admul lec 2251 , espesante carboxi metil celulosa de sodio, saborizante chocolate 04285, saborizante chocolate 51895 .

Investigación y Desarrollo de Producto. El trabajo de investigación se realizó con granos de procedencia Quillabamba y Valle del Río Apurímac. Se procesó el grano hasta obtener el licor de cacao; éste se usó como materia prima para la elaboración de 
chocolate amargo para taza. Adicionalmente, se emplearon una serie de ingredientes y aditivos alimentarios.

El chocolate amargo para taza fue hecho en un lote de $1000 \mathrm{~g}$. El chocolate fue formulado conteniendo licor de cacao (con dos niveles de tostado, identificados como licor de cacao 1 y 2), café tostado molido y refinado desde $0 \%$ hasta $0.5 \%$, los emulsificantes y el carboxi metil celulosa de sodio se mantuvieron constantes en todas las formulaciones, los espumantes se añadieron desde $0 \%$ hasta $5 \%$ peso/peso (hyfoama DSN y vana lata 30B), el vana capa $23 \mathrm{P}$ desde $5 \%$ hasta $12,80 \%$ y $0.30 \%$ peso/peso de saborizante chocolate de diferentes procedencias Perú y Colombia. Las formulaciones se encuentran detalladas en la tabla 1.

Los licores de cacao de Cusco y Ayacucho fueron derretidos en un horno microondas y mezclados $(50 / 50)$ usando una batidora semi industrial a baja velocidad por 6 horas a $60^{\circ} \mathrm{C}$.

El café tostado molido fue refinado en un refinador de 3 rodillos horizontales a un diámetro de partícula media, para luego ser mezclado con el resto de los ingredientes: espumante, emulsificante y carboxi metil celulosa de sodio a $45^{\circ} \mathrm{C}$ por 10 minutos.

Los chocolates fueron conchados a $60^{\circ} \mathrm{C}$ por 4 horas en la batidora. La lecitina $(0,2 \%)$ fue añadida al comienzo del concheo, el resto fue añadido luego de 2 horas de concheo. Una hora antes que termine el proceso, se añadió el saborizante chocolate. La toma de muestra para la determinación de la viscosidad fue hecha el mismo día.

El chocolate amargo para taza es temperado manualmente con ayuda de una mesa de mármol, termómetro y cronómetro. El chocolate fue temperado siguiendo el perfil tiempo y temperatura para chocolates amargos.

Las muestras fueron empacadas en papel aluminio y almacenadas a $20^{\circ} \mathrm{C}$ por 30 días previos a los análisis.

\section{Análisis físico químico}

Determinación de la cantidad de espuma en la taza. Se realizó de acuerdo al siguiente método:

En una licuadora se colocó $200 \mathrm{~mL}$ de agua caliente, $16 \mathrm{~g}$ de azúcar y $8 \mathrm{~g}$ de chocolate amargo para taza, y se licuó enérgicamente por 1 minuto, se añadió en probeta de $250 \mathrm{~mL}$ y se midió la cantidad de espuma en volumen, al tiempo inicial (inmediatamente después del vertido) y luego de 10 minutos.

Determinación de la cantidad de grasa, humedad, contenido de sólidos grasos y composición de ácidos grasos. Estas características se miden de acuerdo a los siguientes métodos oficiales:

Determinación de la cantidad de grasa: método 963.15 AOAC (1995), método de extracción por Soxhlet (1). Determinación de humedad: método 931.04 AOAC (1995) (1). Determinación del contenido de sólidos grasos: método Cd 16b - 99 (99) AOCS (1997) (2). Determinación de la composición de ácidos grasos: método Ce 1-62 (97) AOCS (1997) (2).

Evaluación sensorial. Se utilizó la prueba de análisis descriptivo (ordenamiento); para lo cual se contó con panelistas entrenados. El director de la prueba elaboró una hoja de calificación para un ordenamiento de puntuación, en el cual se aplica una puntuación que refleja razones o proporciones de intensidad. A la muestra patrón se le asigna un va-

Tabla 1. Fórmulas para las alternativas DP-006 a la DP-013.

\begin{tabular}{|c|c|c|c|c|c|c|c|c|}
\hline Ingredientes & DP - 006 & DP - 007 & DP - 008 & DP - 009 & DP - 010 & a) 10 & DP - 012 & DP - 013 \\
\hline Licor de Cacao 2 & $96.30 \%$ & $96.30 \%$ & $95.30 \%$ & $95.30 \%$ & $94.30 \%$ & & $93.30 \%$ & $85.50 \%$ \\
\hline Hyfoama DSN & $0.00 \%$ & $2.00 \%$ & $0.00 \%$ & $3.00 \%$ & $4.00 \%$ & & $0.00 \%$ & $0.00 \%$ \\
\hline Vana Capa $23 \mathrm{P}$ & $0.00 \%$ & $0.00 \%$ & $0.00 \%$ & $0.00 \%$ & $0.00 \%$ & 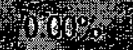 & $5.00 \%$ & $12.80 \%$ \\
\hline Vana Lata $30 \mathrm{~B}$ & $2.00 \%$ & $0.00 \%$ & $3.00 \%$ & $0.00 \%$ & $0.00 \%$ & $0.00 \%$ & $0.00 \%$ & $0.00 \%$ \\
\hline Café Tostado Molido & $0.50 \%$ & $0.50 \%$ & $0.50 \%$ & $0.50 \%$ & $0.50 \%$ & & $0.50 \%$ & $0.50 \%$ \\
\hline Lecitina de Soya & $0.30 \%$ & $0.30 \%$ & $0.30 \%$ & $0.30 \%$ & $0.30 \%$ & 8 & $0.30 \%$ & $0.30 \%$ \\
\hline Emulsificante: Admul Lec 2251 & $0.30 \%$ & $0.30 \%$ & $0.30 \%$ & $0.30 \%$ & $0.30 \%$ & & $0.30 \%$ & $0.30 \%$ \\
\hline Carboxi Metil Celulosa de Sodio & $0.30 \%$ & $0.30 \%$ & $0.30 \%$ & $0.30 \%$ & $0.30 \%$ & & $0.30 \%$ & $0.30 \%$ \\
\hline Saborizante Chocolate 51895 & $0.30 \%$ & $0.30 \%$ & $0.30 \%$ & $0.30 \%$ & $0.30 \%$ & & $0.30 \%$ & $0.30 \%$ \\
\hline Total & $100.00 \%$ & $100.00 \%$ & $100.00 \%$ & $100.00 \%$ & $100.00 \%$ & Toosthos & $100.00 \%$ & $100.00 \%$ \\
\hline
\end{tabular}


lor numérico cualquiera, a libre elección del juez, no siendo cero ni valor negativo. Las demás muestras recibirán una calificación que estará en proporción al valor asignado al patrón y que serán valores múltiplos o submúltiplos de éste. Es conveniente recordar que las muestras preparadas (según el método determinación de la cantidad de espuma en taza), no deben contener leche para no interferir en otros sabores. El análisis estadístico paramétrico que validará el análisis sensorial se efectuará a las razones entre calificaciones después de su normalización, con la prueba $\mathrm{F}$ y con un diseño de bloques completos al azar (D.B.C.A.).

Estabilidad. La metodología (Subramaniam, 1999) es la siguiente:

Una vez obtenido el producto, éste se coloca sin empacar en placas Petri cubiertas, la evaluación dura aproximadamente 12 semanas, se requiere 84 unidades de muestras, cada una $100 \mathrm{~g}$. La mitad de las muestras se colocan (por triplicado) en una cámara o ambiente a temperaturas cíclicas cambiantes de 17 ${ }^{\circ} \mathrm{C}$ por 12 horas a $27^{\circ} \mathrm{C}$ por otras 12 horas adicionales, todo a $50-60 \%$ HR., por tres semanas consecutivas. En la cuarta semana se deja el producto a $20^{\circ} \mathrm{C}, 50$ $60 \%$ HR por toda la semana. Se repite la evaluación hasta la $12^{\text {va }}$ semana. Se realiza la evaluación sensorial desde la primera semana (una evaluación por semana) de sometido el producto a las pruebas aceleradas y por cada semana que transcurre (cuadro 3). Paralelamente se evalúa un set de muestras que representan el blanco ( $50 \%$ de las muestras), éstas no son sometidas a condiciones aceleradas, de esta manera se obtiene una mejor evaluación sensorial del producto. Cuando alguna de sus características disminuyan en intensidad, ahí es donde se delimita el tiempo de vida del producto.

Prueba industrial. La prueba industrial se realiza en dos plantas procesadoras. Con la formulación de la tabla 1, se procede a realizar la prueba con una cantidad de $1000 \mathrm{~kg}$.

\section{RESULTADOS}

Investigación y desarrollo de producto. Se evaluaron las muestras con respecto al patrón (tabla 2) y se empezó a evaluar sensorialmente con las cinco primeras fórmulas (tabla 1) con el licor de cacao 1, 2 y el café tostado molido, los resultados de los jueces indican que es necesario la elaboración de otras
Tabla 2. Características físico químicas del chocolate para taza amargo.

\begin{tabular}{ll}
\hline Características & Resultados \\
\hline$\%$ Grasa & 55.00 \\
\% Humedad & 2.05 \\
Contenido de Sólidos Grasos & \\
$10^{\circ} \mathrm{C}$ & 89.60 \\
$20^{\circ} \mathrm{C}$ & 65.10 \\
$25^{\circ} \mathrm{C}$ & 23.40 \\
$30^{\circ} \mathrm{C}$ & 1.10 \\
$35{ }^{\circ} \mathrm{C}$ & 0.40 \\
$37^{\circ} \mathrm{C}$ & 0.30 \\
Composición de Acidos Grasos & \\
C:16:0 & 27.20 \\
C:16:1 & 0.70 \\
C:18:0 & 30.10 \\
C:18:1 & 36.90 \\
C:18:2 & 3.40 \\
C:18:3 & 0.20 \\
C:20:0 & 0.90 \\
Vol:0 & 0.20 \\
Inicial (ml) & \\
Final (ml) & 38 \\
\hline
\end{tabular}

muestras. La segunda vez se presentó otras alternativas como se indican en la tabla 1 con el licor de cacao 2 y los resultados de los jueces indican que se llegó a la muestra ideal (tabla 1, DP - 011). El incremento de la temperatura en el tostado de $140^{\circ} \mathrm{C}$ a $160^{\circ} \mathrm{C}$ hizo incrementar el color marrón del licor de cacao, haciéndolo muy cercano al patrón.

Análisis físico químico. Se evaluó la cantidad de espuma del producto, tanto inmediatamente después de servir en taza como luego de transcurridos 10 minutos. Como se observa, la DP - 011 es la que presenta mejores características de espuma en taza en el lapso de 10 minutos (tabla 3 ).

Estabilidad. Una vez obtenido el producto ideal, es colocado sin empaque, en las placas petri, para empezar a realizar la prueba de estabilidad, contando para ello con ayuda de la metodología de Subramaniam (6). Siguiendo la metodología del trabajo, se observa que el producto tiene un tiempo de vida de 10 meses a condiciones especiales $18-20{ }^{\circ} \mathrm{C}$ y $50-60 \% \mathrm{HR}$.

Prueba industrial. Obtenida la muestra ideal y validado el tiempo de vida del producto, se procedió 
Tabla 3. Contenido de grasa, humedad contenido de solidos grasos y composición de ácidos grasos del chocolate para taza amargo DP-011 (Mejorado).

\begin{tabular}{lc}
\hline Determinaciones & Resultados \\
\hline$\%$ Grasa & 48.80 \\
\% Humedad & 0.88 \\
Contenido de Sólidos Grasos & \\
$10^{\circ} \mathrm{C}$ & 88.10 \\
$20^{\circ} \mathrm{C}$ & 61.10 \\
$25^{\circ} \mathrm{C}$ & 17.50 \\
$30^{\circ} \mathrm{C}$ & 1.00 \\
$35^{\circ} \mathrm{C}$ & 0.30 \\
$37^{\circ} \mathrm{C}$ & 0.30 \\
Composición de Ácidos Grasos & \\
C:16:0 & 27.70 \\
C:16:1 & 0.30 \\
C:18:0 & 30.70 \\
C:18:1 & 35.90 \\
C:18:2 & 3.50 \\
C:18:3 & 0.10 \\
C:20:0 & 0.90 \\
C:2:0 & 0.20 \\
\hline
\end{tabular}

a la prueba industrial considerando un lote de 1000 $\mathrm{kg}$, con la finalidad de obtener todos los procesos bajo control, definir los parámetros de trabajo, evaluar rendimientos y costos reales.

Discusión. Como se observa en la tabla 2, donde se muestran las características físico químicas del patrón, éste tiene una cantidad de grasa de $55 \%$, cuyo valor está dentro de los requerimientos para chocolate amargo en la Norma Técnica Peruana, las otras características, tales como: humedad, contenido de sólidos grasos, composición de ácidos grasos y volumen de espuma son propiedades para determinar las características internas del producto. El contenido de sólidos grasos indica que el chocolate se derrite fácilmente a la temperatura corporal $\left(37^{\circ} \mathrm{C}\right)$ ya que desde $\operatorname{los} 30^{\circ} \mathrm{C}$ el contenido de sólidos grasos va bajando desde $1,10 \%$ hasta $0,30 \%$. La composición de ácidos grasos indica la presencia de dichos ácidos en cl chocolate amargo para taza, provenientes de la manteca de cacao. El volumen de espuma se ha determinado en una preparación en taza, notándose una gran cantidad de espuma para la DP - 011 (tabla 1) por la presencia de proteínas en su composición.

El la evaluación de inicio, el efecto del sabor y aroma a amargo utilizando café tostado molido en la formulación se observa que a mayores incrementos hasta un $0,50 \%$ tenemos resultados satisfactorios, pero también el efecto de un mayor nivel de tostado en el grano de cacao ofrece un sabor amargo mayor, característica del patrón.

En la tabla 1 se indican formulas que son usadas con el licor de cacao 2, es decir, con el tostado de 160 ${ }^{\circ} \mathrm{C}$ x 40 minutos, reflejando los resultados en que el color se incrementó, el aroma y sabor por el uso de saborizante chocolate de diferente procedencia. De las 8 fórmulas se escogieron para evaluación sensorial la DP - 003, DP - 004 (ambos por el contenido diferente de café tostado molido a un mismo tipo y nivel de saborizante chocolate), DP - 007 (por tener un saborizante diferente a un mismo nivel de café tostado molido) y DP - 011 (cuyos resultados en contenido de espuma son parecidos al patrón).

Se sabe que a partir del propio licor de cacao peruano no es posible obtener el mismo perfil de sabor a chocolate amargo para taza, o con tan sólo con la mezcla de licores de cacao (50\% Ayacucho y $50 \%$ Cusco), por ello se determinó trabajar con saborizantes chocolate de una compañía colombiana y una peruana.

De igual forma, se decidió añadir agentes espumántes, ya que el producto original (patrón) al servirlo en taza presenta una cantidad de espuma similar a la preparación de un capuchino.

Se ha evaluado también la cantidad de espuma, definiendo la cantidad de espuma al volumen que ocupa en la probeta y la permanencia de éste durante un lapso (tiempo en que se espera que el consumidor ya haya terminado todo el chocolate para taza amargo), en este ensayo se determinó un tiempo de 10 minutos. También se puede apreciar que se han usado tres tipos de espumantes. Concluyendo que el insumo hyfoama DSN, es el espumante ideal, porque otorga al producto final características similares al patrón, además de su compatibilidad con productos como café y cacao.

Se puede apreciar que ya no existen diferencias entre las muestras debido al trabajo que se realizó con el grano de cacao, en el procesamiento y la formulación. Se han escogido los mejores (calidad sensorial) granos de cacao, se ha incidido en la temperatura de tostado $\left(140\right.$ a $\left.160^{\circ} \mathrm{C}\right)$, reflejando que el color marrón del licor de cacao ha subido el puntaje en la evaluación por los jueces. Además que se han con- 
seguido los mejores saborizantes chocolate, lo cual resultó en beneficio para el presente proyecto, pues el saborizante es el que dio mejores puntajes comparado con el saborizante nacional.

La tabla 3 muestra los resultados de análisis de la fórmula mejorada, se consideró como parámetros de medición: cantidad de grasa, humedad, contenido de sólidos grasos y composición de ácidos grasos.

En la hoja de evaluación del tiempo de vida del chocolate amargo para taza (DP - 011), donde se han evaluado las características del producto indica que el chocolate tiene el "snap" (sonido particular al quiebre del producto) hasta las 9 semanas, la migración de grasa se presenta a las 11 semanas, el chocolate no presenta enranciamiento durante su

Figura 1. Resultados de la Evaluación de la Espuma en Muestras.

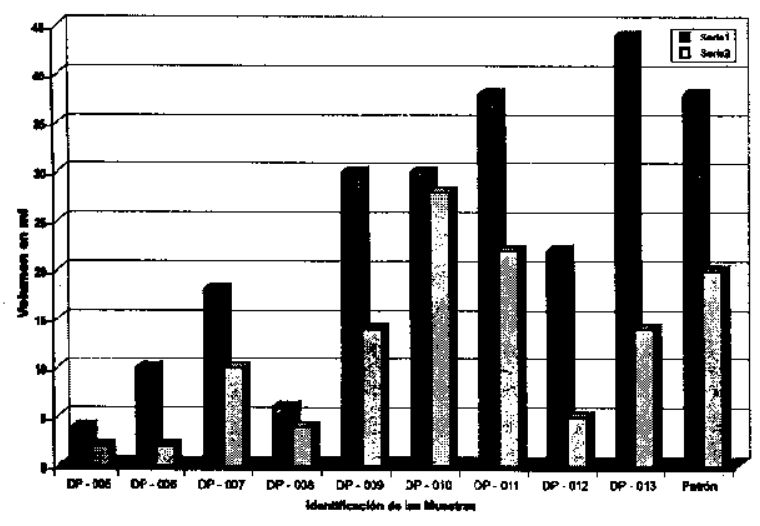

evaluación, el aroma persiste hasta las 10 semanas, la espuma hasta las 11 semanas y el brillo hasta las 9 semanas.

\section{REFERENCIAS BIBLIOGRÁFICAS}

1. AOAC. Official Methods of Analysis of Association of Offitial Agricultural Chemist International. $16^{\text {th }}$ Edition, Volume II. Edited by Patricia Cunniff. Virginia. 1995, p. 1-17.

2. AOCS. Official Methods 2nd recommended practices of the American Oil Chemists Society. 5ta Edition, $1^{\text {st }}$ Printing. 2000 Methods. Champaign, Illinois. 1997.

3. Beckett $\mathbf{S}$ T. Fabricación y utilización industrial del chocolate. Editorial Acribia. S.A. Zaragoza. 1994.

4. NTP 208.002. Chocolate. Requisitos. INDECOPI. $2^{\mathrm{a}}$ ed. Lima. 1999.

5. NTP 208.012. Cacao y Chocolate. Cacao sin cáscara ni germen, cacao en pasta, torta de prensado de cacao y polvillo de cacao (fino de cacao) para uso en la fabricación de productos de cacao y chocolate. INDECOPI. $2^{\text {a }}$ ed. Lima. 1999.

6. Subramaniam P. Accelerated Shelf-Life Testing. The Manufacturing Confectioner, 1999. Glen Rock, NJ, p. 147-152.

7. Ureña $\mathbf{P} \mathbf{M}, D^{\prime}$ Arrigo $\mathrm{H}$ M, Girón, M. O. Evaluación Sensorial de los Alimentos. Universidad Nacional Agraria La Molina. 1ª ed. Lima. 1999. 